\title{
Tumor del estroma extra gastrointestinal (E-GIST) gigante de omento*
}

\author{
Drs. RENÉ GORDILLO V. ${ }^{1}$, JOSÉ BELTRÁN N. ${ }^{2}$, MIRIAM DÍAZ³
}

\author{
Servicio de Cirugía General. \\ Servicio de Anatomía Patológica del Hospital del Instituto Ecuatoriano de Seguridad Social de Ibarra. \\ 3 Servicio de Anatomía Patológica, Hospital "Carlos Andrade Marín”, Quito. \\ Ecuador.
}

\begin{abstract}
Extra gastrointestinal stromal tumor (E-GIST) of the omentum

Introduction: Tumors that are pathologically and immunohistochemically similar to gastrointestinal stromal tumors (GIST), but coming from soft tissues of the mesentery or retroperitoneum are called extra gastrointestinal stromal tumors (E-GISTs) and are more aggressive than GISTs. Clinical case: We report a 46 years old woman operated for a uterine sarcoma with a liver metastasis of the tumor, subsequently subjected to radiotherapy. A new CAT scan showed a $21 \times 12 \mathrm{~cm}$ tumor. Radiotherapy was discontinued and the patient refused chemotherapy. Four years later, the patient consulted in the emergency room for a partial bowel obstruction. A new CAT scan showed a tumor that occupied the abdominal and pelvic cavity. The patient was operated, finding a highly vascularized and multisystem tumor that was fused together with the omentum, which was excised. The pathological diagnosis of the surgical piece was a high risk GIST.
\end{abstract}

Key words: Gastrointestinal stromal tumor, sarcoma, omentum.

\section{Resumen}

Introducción: Los tumores del estroma gastrointestinal (GIST) fueron clasificados inicialmente dentro de otros grupos de tumores (leiomioma, leiomioblastoma, leiomiosarcoma), llamándolos leiomiomas bizarros o leiomiomas celulares debido a su apariencia histológica similar. Con el advenimiento de la microscopia electrónica se demostró que sólo algunos de estos tumores presentaban evidencias ultraestructurales de diferenciación muscular lisa. Posteriormente, los avances en la biología molecular y la inmunohistoquímica han permitido diferenciarlos de otras neoplasias digestivas e identificarlos como una patología clínica e histopatológica propia. Los tumores que histopatológicamente e inmunohistoquímicamente son similares a los GIST, pero proceden de los tejidos blandos del mesenterio o el retroperitoneo sin presentar conexión,

*Recibido el 03 de julio de 2012 y aceptado para publicación el 24 de noviembre de 2012.

Los autores dejan constancia de que no existe ningún conflicto de interés entre ellos, el contenido del artículo ni con ninguna empresa relacionada o no con la salud.

Correspondencia: Dr. René Gordillo V.

Calle Cesar Morales 4-76 y Salvador Dalí, Urbanización Flota Imbabura. Ibarra-Ecuador. rygordillo@hotmail.com 
ni siquiera tenue, con la pared ni con la superficie serosa de las vísceras, son denominanados tumores del estroma extragastrointestinal (E-GIST) y han mostrado tener un comportamiento más agresivo que los GIST originados a nivel gástrico y similar a los intestinales. Reporte del caso: Paciente de 47 años quien desarrolló durante 8 años un tumor abdominal, que resultó ser un tumor del estroma gastrointestinal de omento, sometida a tratamiento quirúrgico. En 18 meses no ha presentado recidiva tumoral.

Palabras clave: GIST, Tumores del estroma extragastrointestinal, c-kit, células intersticiales de Cajal, imatinib.

\section{Caso clínico}

Paciente femenino de 47 años de edad, soltera, menarquia a los 18 años, ciclos regulares de $28 \times 3$ días, G0, P0, A0. No anticoncepción oral ni sistémica, cuya patología inició en mayo de 2003, con dolor pélvico y sangrado vaginal, en su investigación se le realizó un ultrasonido pélvico, que mostró miomas intramurales + una tumoración en endometrio, luego de ello se le realizó un legrado diagnóstico que reportó endometrio mucosecretor, por ello el 10 de noviembre de 2003 fue sometida a histerectomía abdominal simple. El estudio histopatológico reveló sarcoma del estroma de alto grado de malignidad, que ocupaba toda la cavidad uterina, mide $5,5 \times 5$ $\mathrm{cm}$; infiltraba al miometrio en $1,2 \mathrm{~cm}$. No invadía el endocervix, no tenía permeabilidad vascular.

Con este diagnóstico fue enviada al servicio de oncología clínica del hospital de referencia, catalogado como un sarcoma uterino tratado con citoreducción sin estudio ganglionar, estadio II por ser G3. $\mathrm{T} 2$ mayor a $5 \mathrm{~cm}$ para la AJCC.

En estudios de extensión la TC mostró una tumoración hepática de la que se tomó una biopsia dirigida.

Decidieron iniciar radioterapia. El 15 de diciembre de 2003 realizaron cobaltografías en AP y L, se administró en 3 sesiones 1.800 de los 5.000 cGy programados.

La biopsia hepática resultó positiva para metástasis del sarcoma uterino. En una nueva tomografía realizada en enero de 2004 apareció una tumoración de $21 \times 12 \mathrm{~cm}$, la paciente presentaba dolor epigástrico, dispepsia y refería el crecimiento rápido de su abdomen. Por ello se suspendió la radioterapia y se planeó pasar a quimioterapia con 2 ciclos de filgastrin, ciclofosfamida y doxirrubicina, explicándole su pronóstico sombrío. La paciente no aceptó el tratamiento y lo abandonó, perdiéndose contacto con ella.

En diciembre de 2006 regresó al servicio de oncología clínica, en buena condición general con su abdomen distendido y tenso. Valorada por Cirugía General, se decidió no realizar tratamiento quirúrgico, proponiéndole nuevamente el tratamiento quimioterápico, mismo que no aceptó y pierde nuevamente contacto médico. Durante este tiempo la paciente presenta cada vez más distensión abdominal, con compromiso de su condición general y nutricional.

En noviembre de 2010 acudió al servicio de Emergencias del Hospital del IESS de Ibarra, presentando por primera ocasión un cuadro obstructivo parcial de 2 días de evolución. A su llegada y valoración inicial se encuentra un abdomen globoso, con una tumoración que ocupa toda la cavidad abdominal. Fue ingresada para manejo clínico y estudio de su patología.

La TC mostró una tumoración multiquística muy vascularizada que ocupaba la cavidad abdominopélvica y que desplaza las vísceras, sin signos de invasión a otros órganos (Figura 1 a, b y c).

Marcadores tumorales, CA 125: 113 y el CA 19-9: 4,48. Las imágenes del tórax no mostraron alteración.

El 10 de diciembre de 2010 fue sometida a laparotomía exploratoria. Al abordaje encontramos una tumoración que aparentaba estar totalmente cubierta de omento, densamente fusionada con él, era una formación multiquística muy vascularizada, dependiente del omento. Luego no se evidenció afectación de ninguna víscera abdominal o pélvica ni rastros de implantes en las superficies serosas o adenopatías (Figuras 2 y 3 a y $3 b$ ).

El tumor se resecó íntegro, con un diámetro mayor de $47 \mathrm{~cm}$ y con un peso de $14,5 \mathrm{~kg}$. Siendo el tumor de mayor tamaño y peso documentado en la literatura de nuestro país (Figura 4).

La paciente se recuperó excelentemente, fue dada de alta a los 4 días. Sin rastro de actividad tumoral 18 meses luego de la cirugía.

El estudio histopatológico reportó: Neoplasia constituida por proliferación de células fusiformes en fascículos, con citoplasmas amplios y núcleos de bordes afilados, algunas células muestran multinucleación, ausencia de mitosis y baja tasa de proliferación celular.

Patente de crecimiento: Infiltrativa.

Patente histológica: Fusocelular.

El estudio Inmunohistoquímico demostró:

Vimentina: Inmunomarcaje positivo, intenso en células neoplásicas.

Actina: Inmunomarcaje positivo, débil en células neoplásicas. 

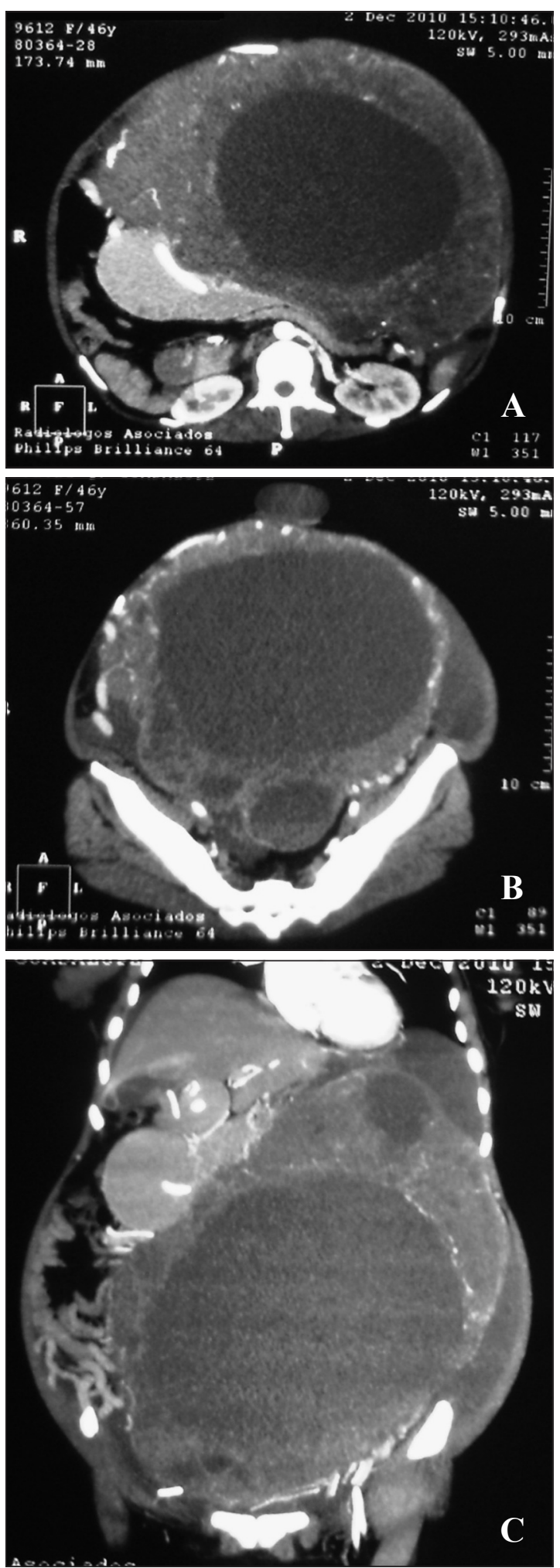

Figura 1 A, B y C. Tomografía axial computada de abdomen.

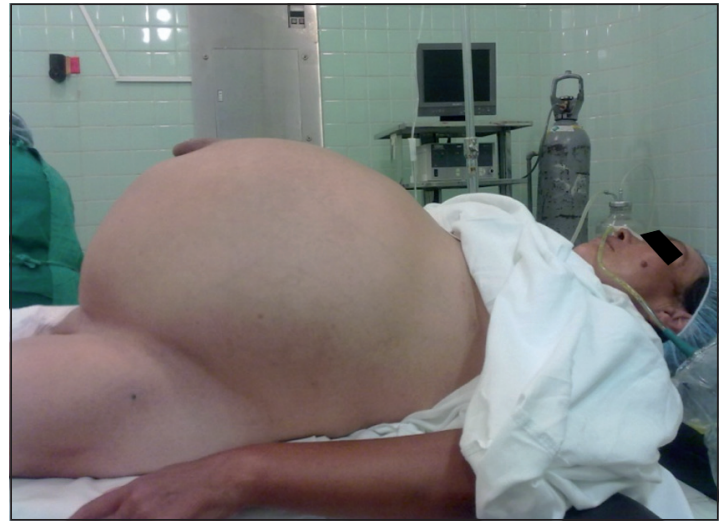

Figura 2. La paciente momentos previos a la inducción anestésica.
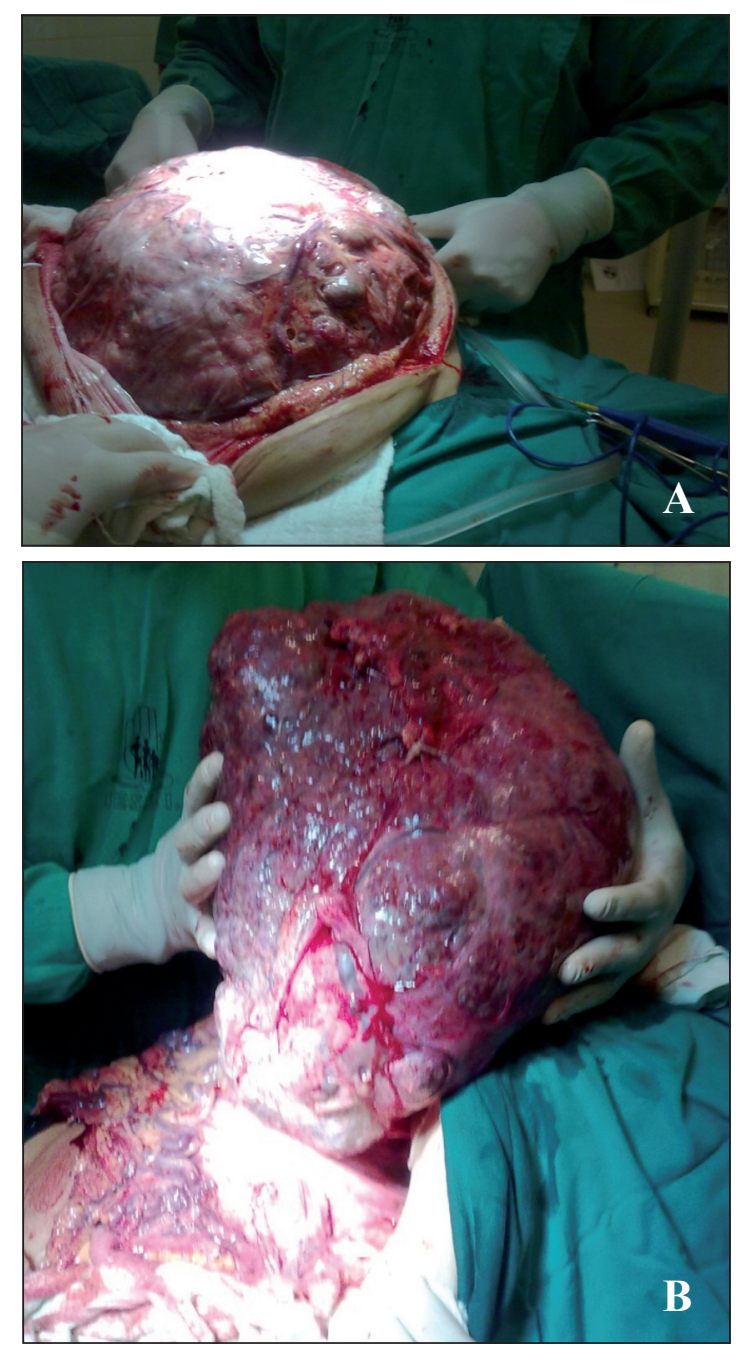

Figura 3. Aspecto intraoperatorio de la tumoración: A y B. 
AEM: Inmunomarcaje focalmente positivo.

S 100: Inmunomarcaje negativo.

Ki 67: Inmunomarcaje negativo.

CD34: Inmunomarcaje positivo, intenso en células neoplásicas.

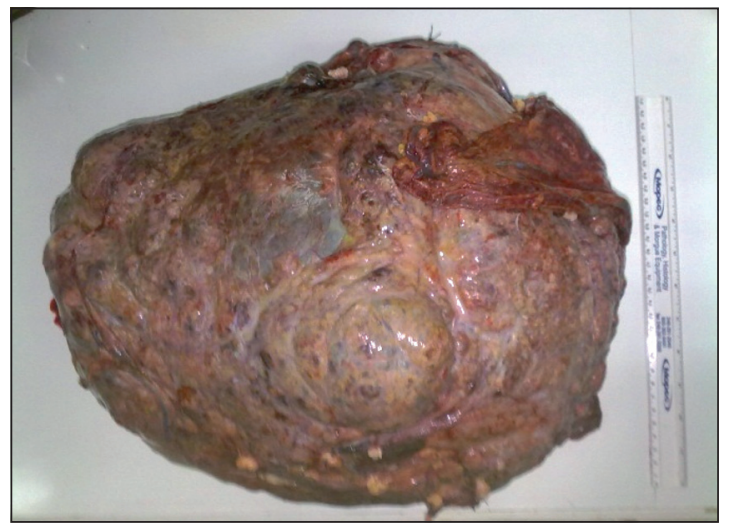

Figura 4. Aspecto ex vivo de la tumoración y su medición.

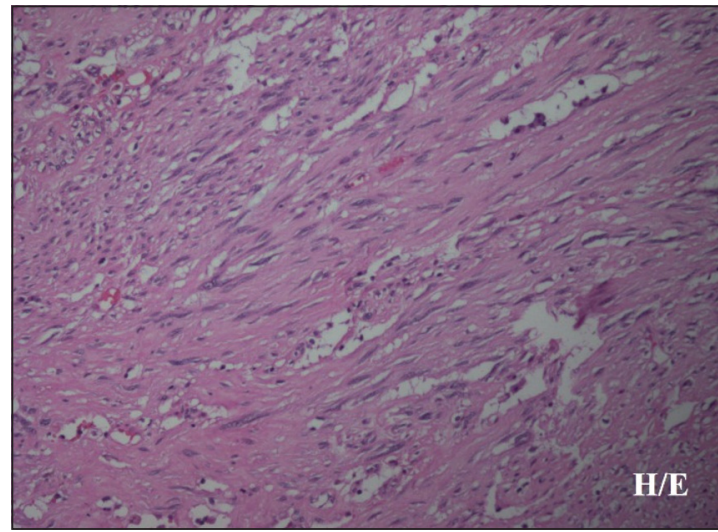

Figura 5. H\&E, 200X, detalle del patrón fusocelular.

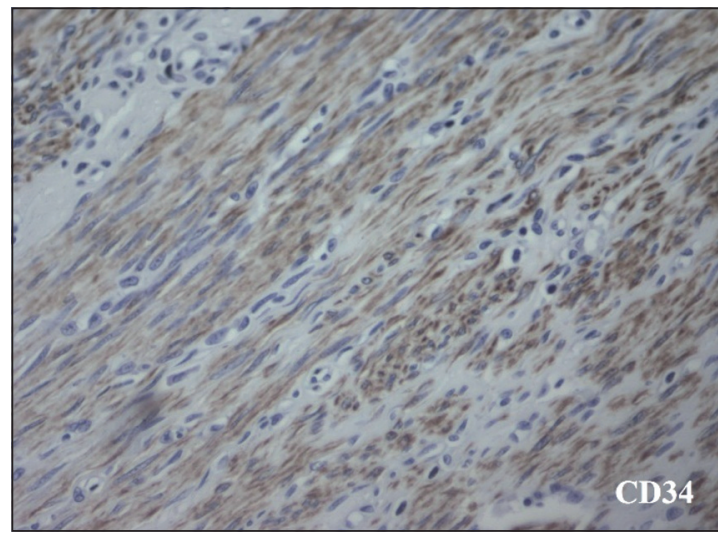

Figura 7. CD34, 400X, inmunoexpresión CD34, positivo en células tumorales.
CD 117: Inmunomarcaje positivo, intenso en células neoplásicas.

Conclusión: Tumor estromal del aparato digestivo GIST, de alto riesgo (Figuras 5, 6, 7 y 8).

\section{Discusión}

Las primeras descripciones de tumores mesenquimáticos del tracto gastrointestinal datan de 1941. Durante años se incluyeron dentro del grupo de tumores derivados de células musculares lisas basados en su parecido histológico, por ello fueron llamados leiomiomas bizarros, leiomiomas celulares y leiomiosarcomas ${ }^{1,2}$. En 1960, Stout propuso el término leiomioblastoma para lesiones gastrointestinales con una apariencia epiteloide o de células redondeadas y sugirió su origen en células inmaduras de músculo liso, esta descripción causaba mucha confusión.

En 1983, Mazur y Clark, documentaron la ausencia de marcadores inmunohistoquímicos para

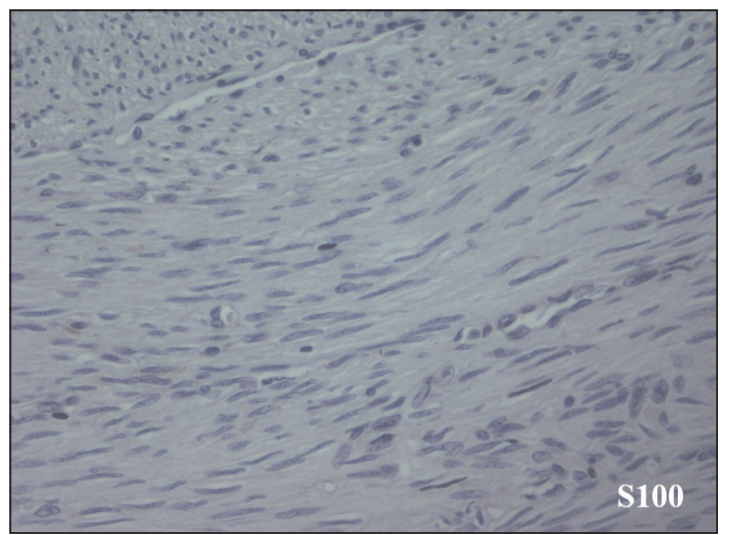

Figura 6. S100, 400X, Inmunoexpresión S100, negativa en células tumorales.

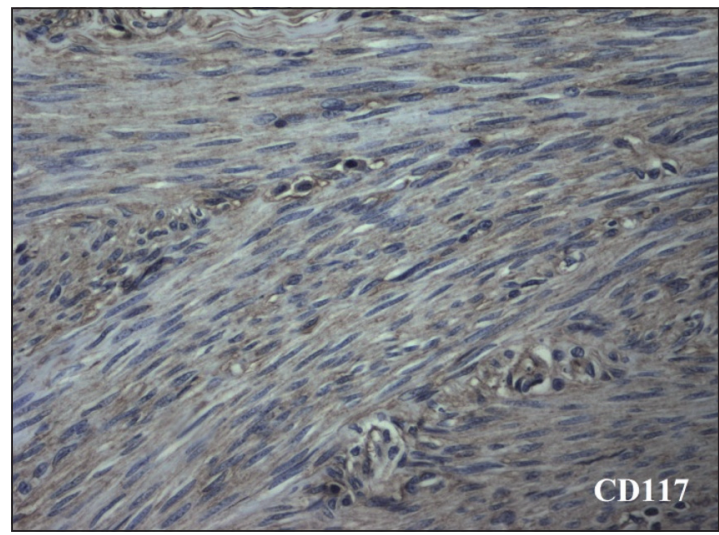

Figura 8. CD117, 400X, inmunoexpresión CD117, positivo en células tumorales. 
músculo en la mayoría de estas lesiones, así como el hallazgo inesperado de marcadores neurales en algunos de ellos. Postularon entonces que estas lesiones mostraban varias líneas de diferenciación mostrando elementos de la pared intestinal y propusieron el término gastrointestinal stromal tumor (GIST) ${ }^{3}$.

En 1984, Herrera y cols describieron una diferenciación neuronal autonómica en algunos subtipos de este tumor.

En 1998, Hirota descubrió mutaciones funcionantes en el protooncogén C-kit, que puede ser detectada con el anticuerpo monoclonal CD-117 ${ }^{4}$.

Los GIST se originan a partir de las células intersticiales de Cajal, o desde células precursoras primitivas que se diferencian hacia células intersticiales de Cajal y hacia células fenotípicamente musculares lisas.

Las células intersticiales de Cajal se encuentran intercaladas entre los nervios autonómicos y las células musculares lisas (plexos mientéricos). Generan el ritmo autónomo de contracciones involucradas en la digestión y peristaltismo, por ello también son llamadas células marcapaso del tracto gastrointestinal.

Los GIST son un tipo infrecuente de tumor de origen mesenquimático, estimándose que corresponden al $0,2 \%$ de los tumores gastrointestinales, es el más común de los sarcomas del tracto gastrointestinal representando el 5\% de todos ellos 5 . Tiene una incidencia aproximada de entre 10 y 20 por millón, la cual es constante en las distintas áreas geográficas y etnias. Aparecen predominantemente en adultos y ancianos cuya mediana oscila entre 55 y 65 años, encontrándose en adultos menores de 40 años entre el 5 y $20 \%$ de los casos y en niños en menos del $3 \%{ }^{6}$.

No existe diferencias entre sexos, excepto en pacientes portadores de la triada de Carney (GIST gástrico maligno, condroma pulmonar y paraganglioma extra adrenal funcionante), que ocurre más frecuentemente en mujeres. No tienen factores de riesgo conocidos, sin embargo, parece haber una influencia genética que se aprecia en reportes de GIST familiares y otros asociados a la enfermedad de von Recklinghausen.

Son heterogéneos respecto a morfología, tamaño y conducta biológica, variando desde tumores con comportamiento benigno hasta tumores muy agresivos y metastásicos al momento de su diagnóstico.

Macroscópicamente son tumores bien circunscritos, flexibles, de color rosa pálido, frecuentemente muestran hemorragia, necrosis y degeneración quística.

Microscópicamente se dividen en 3 diferentes subgrupos histológicos: fusocelular $70 \%$, epiteloide $20 \%$ y mixtos $10 \%$, sin que se haya probado que influya sobre el pronóstico.
Se han descrito con una gran variación de tamaño, desde tumores de menos de $1 \mathrm{~cm}$ a lesiones muy grandes de hasta $35 \mathrm{~cm}$ con una media de $5 \mathrm{~cm}^{5}$. En el presente caso el tumor alcanzó $47 \mathrm{~cm}$ de diámetro mayor y $14,5 \mathrm{~kg}$ por lo cual resulta ser el E-GIST de mayor tamaño reportado en la literatura mundial hasta la fecha.

Independientemente del tamaño tumoral, los GIST comparten características morfológicas y de inmunoreactividad. Su clave molecular es la proteína transmembrana KIT (CD117) localizada en el brazo largo del cromosoma 11 (4q11-12).

Inmunohistoquímicamente, $60-70 \%$ de los GIST expresan positividad para CD $34,30-40 \%$ son positivos para actina de músculo liso, $5-10 \%$ son positivos para el antígeno de la proteína S-100. Sin embargo, ninguno de ellos es específico para diagnosticar GIST en ausencia de positividad para KIT. La expresión de la proteína KIT puede ser detectada con el anticuerpo monoclonal CD-117, con esta técnica, se diagnostican el 94-95\% de los GIST ${ }^{7}$.

Aunque podrían localizarse en cualquier sitio del tracto gastrointestinal, más del $50 \%$ de los casos se presentan a nivel gástrico ${ }^{8}$. No obstante, se han descrito en ubicaciones extragastrointestinales como mesenterio y omento, retroperitoneo, vesícula biliar, pared vesical, ovario y útero ${ }^{9-16}$ y sincrónicamente con otros tumores gastrointestinales ${ }^{17,18}$.

El índice mitótico, el tamaño tumoral y el sitio del tumor son los más importantes predictores de metástasis.

Índices mitóticos mayores a $>2 / 50 \mathrm{HPF}$, presencia de necrosis y alta celularidad describen un comportamiento más agresivo de los EGIST ${ }^{19-22}$.

\section{Tratamiento}

La resección quirúrgica es el gold standard, su objetivo es la resección total de la lesión sin romper el tumor por el riesgo de desarrollar implantes en las superficies peritoneales. La supervivencia está determinada por el tamaño tumoral y no por los márgenes microscópicos negativos. No requiere linfadenectomía regional ya que sólo en raras ocasiones invaden a los ganglios. La resección quirúrgica completa asegura una supervivencia a 5 años de 48 a $65 \%{ }^{23-25}$.

\section{Conclusión}

En los últimos años se ha adquirido un gran conocimiento acerca del diagnóstico y tratamiento de esta patología, que aún infrecuente, debe permanecer en mente al momento de realizar un diagnóstico diferencial de las lesiones del tracto gastrointestinal. 


\section{Referencias}

1. Muñoz C, Sabah S, Navarro A, Planzer M, Silva C, Santander R. Tumores del estroma gastrointestinal (GIST): Revisión de la literatura. Gastr Latinoam. 2006;17:4351.

2. Bórquez P, Neveu R. Tumores del estroma gastrointestinal (GIST), un particular tipo de neoplasia. Rev Med Chile 2008;136:921-9.

3. Miettinen M, Lasota J. Gastrointestinal Stromal Tumors. Review on Morphology, Molecular Pathology, Prognosis, and Differential Diagnosis. Arch Pathol Lab Med. 2006;130:1466-78.

4. Yoon-Koo K, Kyoung-Mee K, Taesung S, Dongil C, Hye Jin K, Min-Hee R, et al. Clinical Practice Guideline for Accurate Diagnosis and Effective Treatment of Gastrointestinal Stromal Tumor in Korea. J Korean Med Sci. 2010;25:1543-1552 doi: 0.3346/jkms. 2010.25.11.1543.

5. Reith J, Goldblum J, Lyles R, Weiss S. Extragastrointestinal (Soft Tissue) Stromal Tumors: An Analysis of 48 Cases with Emphasis on Histologic Predictors of Outcome. Modern Pathology 2000;13:577-85.

6. Castillo-Sang M, Mancho S, Tsang A, Gociman B, Almaroof B, Ahmed M. A malignant omental extragastrointestinal stromal tumor on a young man: a case report and review of the literature. World J of Surg Oncol. 2008;6:50 doi:10.1186/1477-7819-6-50.

7. Weiss S, Goldblum J. En Enzinger y Weiss Tumores de Partes Blandas; 5ta ed. 2009; Elsevier-España; 565-81.

8. Peralta E. Rare Anorectal Neoplasms: Gastrointestinal Stromal Tumor, Carcinoid, and Lymphoma. Clin Colon Rectal Surg. 2009;22:107-14. doi 10.1055/s-00291223842.ISSN 1531-0043.

9. Contreras J, Atienza L, Pérez J, Beltrán M, Baena J. GIST: A propósito de un término confuso y presentación de un caso de tumor del estroma extragastrointestinal. Oncología 2006;29:254-8.

10. Hyo-Cheol K, Jeong-Min L, Se-Hyung K, Kyoung K, Minjin L, Young K, et al. Primary Gastrointestinal Stromal Tumors in the Omentum and Mesentery: CT Findings and Pathologic Correlations. AJR 2004;182:1463-7.

11. Todoroki T, Sano T, Sakurai S, Segawa A, Saitoh T, Fujikawa K, et al. Primary Omental Gastrointestinal Stromal Tumor (GIST). World J of Surg Oncol. 2007;5:66 doi:10.1186/1477-7819-5-66.

12. Rubin BP, Singer S, Tsao C, Duensing A, Lux ML, Ruiz R, et al. KIT Activation Is a Ubiquitous Feature of Gastrointestinal Stromal Tumors. Cancer Res. 2001;61:8118-21.

13. Engin G, Asoglu O, Kapran Y, Mert G. A gastrointestinal stromal tumor with mesenteric and retroperitoneal invasion. World J of Surg Oncol. 2007;5:121 doi:10.1186/1477-7819-5-121.

14. Pauser U, Hinz S, Merz H, Feller A. Gastrointestinal stromal tumors with pseudocystic change mimicking a pancreatic tumor: two case reports. Journal of Medical Case Reports 2009;3:7592 doi: 10.1186/1752-1947-37592.

15. Kyung J, Hye S, Lee S, Ouk-Min K, Seob S, Myung H. Malignant Gastrointestinal Stromal Tumor of the Gallbladder. J Korean Med Sci. 2004;19:763-7.

16. Giorgio C. A gastrointestinal stromal tumor (GIST) masquerading as an ovarian mass. World J of Surg Oncol. 2004;2:15.

17. Samaras V, Foukas P, Triantafyllou K, Leontara V, Tsapralis D, Tsompanidi E, et al. Synchronous well differentiated neuroendocrine tumour and gastrointestinal stromal tumour of the stomach: a case report. BMC Gastroenterology 2011;11:27.

18. Chien-Yuan H, Ming-Jen C, Shou-Chuan S, TsangPai L, Yu-Jan C, Tsang-En W, et al. Gastric carcinoid tumor in a patient with a past history of gastrointestinal stromal tumor of the stomach. World J Gastroenterol. 2008; 14: 6884-7.

19. Takahashi T, Kuwao S, Yanagihara M, Kakita A. A primary solitary tumor of the lesser omentum with immunohistochemical features of gastrointestinal stromal tumors. Am J Gastroenterol. 1998;93:2269-73.

20. Van der Zwan S, De Matteo R. Gastrointestinal Stromal Tumor: 5 Years Later. American Cancer Society 2005;104:1781-8.

21. Mee-Yon C, Jin Hee S, Joon Mee K, Kyoung-Mee K, Young Su P. Current Trends in the Epidemiological and Pathological Characteristics of Gastrointestinal Stromal Tumors in Korea, 2003-2004. J Korean Med Sc.i 2010;25: 853-62.

22. Gourgiotis S, Kotoulas D, Aloizos S, Kolovou A, Salemis N, Kantounakis I. Preoperative diagnosis of obscure gastrointestinal bleeding due to a GIST of the jejunum: a case report. Cases Journal 200; 2:8186. doi: 10.4076/1757-1626-2-8186.

23. Motegi A, Sakurai S, Nakayama H, Sano T, Oyama T, Nakajima T. PKC-theta, a novel immunohistochemical marker for gastrointestinal stromal tumors (GIST), especially useful for identifying KIT-negative tumors. Pathol Int. 2005;55:106-12.

24. Stamatakos M, Douzinas E, Stefanaki C, Safioleas P, Polyzou E, Levidou G, et al. Gastrointestinal stromal tumor. World J of Surg Oncol 2009;7:61. doi: 10.1186/1477-7819-7-61.

25. Di Vizio D, Demichelis F, Simonetti S, Pettinato G, Terracciano L, Tornillo L, et al. Skp2 expression is associated with high risk and elevated Ki67 expression in gastrointestinal stromal tumours. BMC Cancer 2008;8:134. doi:10.1186/1471-2407-8-134. 